\title{
COMPUTABLE ERROR BOUNDS WITH IMPROVED APPLICABILITY CONDITIONS FOR COLLOCATION METHODS
}

\author{
A.H. AHMED \\ Visiting Professor \\ Instituto de Matematica Pura e Aplicada, IMPA \\ Estrada Dona Castorina 110 - Jardim Botanico \\ CEP 22460-320 - Rio de Janeiro - RJ, BRAZIL
}

(Received July 10, 1995 and in revised form February 5, 1997)

\begin{abstract}
This paper is concerned with error bounds for numerical solution of linear ordinary differential equation using collocation method. It is shown that if the differential operator is split in different operator forms then the applicability conditions for the computable error bounds which are based on the collocation matrices could be improved.
\end{abstract}

KEY WORDS AND PHRASES: Error bounds, collocation, differential equations. 1991 AMS SUBJECT CLASSIFICATION CODES: G17.

\section{INTRODUCTION}

This paper extends previous work of Cruickshank \& Wright [1] and Ahmed [2] on computable error bounds for collocation solution of ordinary differential equations. In [1] computable error bounds for the solution with the global collocation method were described in terms of matrices related to the highest derivatives of the solution. It is shown later in [2] that if the bounds were related directly to the matrices involved in the solution and not via the highest derivatives then significant improvement in the closeness of these bounds could be achieved. However, despite this improvement, the conditions of applicability which were the main drawback of [1] have turned out to be the same. That is for many practical problems an inordinate amount of work is necessary to produce any strict error bounds of this type. The aim of this paper is to consider this problem in order to improve the applicability of these bounds. The work in this paper is developed from Ahmed's thesis [3].

\section{THE NEW SPLITTING OF THE DIFFERENTIAL OPERATOR}

Before investigating the result more precisely we introduce the following assumptions and notations.

We consider an $m$-th order differential equation of the form

$$
x^{m}(t)+\sum_{j=0}^{m-1} p_{j}(t) x^{(\jmath)}(t)=y(t)
$$

with associated homogeneous boundary conditions. The theory in [1] and [2] deals with this equation in the operator form

$$
\left(D^{m}-T\right) x=y
$$

where $\left(D^{m} x\right)(t)=\left(\frac{d^{m} x}{d t^{m}}\right)(t)$ But as indicated in Kantorvich \& Akilov [4] and Anselone [5], the theory can be applied to a general equation of the form

$$
(D-T) x=y,
$$

where $D$ is invertible. Clearly different choices may be treated to deal with the problem of applicability. However, there are some practical difficulties which would limit them. Firstly the inverse of $D$ needs to 
be known explicitly. Secondly the procedure for calculating the projection norm or bound on it needs to be available. Thirdly all the assumptions required by the theory in [4] and [5] should be satisfied.

To avoid the difficulty of knowing the inverse of $D$ explicitly, perhaps the simplest extension could be to define the differential operator $D$ by

$$
D x=D^{m} x+\lambda_{m-1} D^{m-1} x+\cdots+\lambda_{0} x
$$

where the $\lambda$ 's are some parameters to be chosen to give the highest possible applicability with a reasonable amount of work. Since $D$ is a linear differential operator with constant coefficients its inverse if it exists can be found analytically.

The norm of the project operator associated with $D$, which will be denoted by $\phi^{*}$ was shown in Ahmed [6] to be asymptotically the same as the norm of the usual interpolating projection operator if, for the global collocation method, the points are more usually Tchebychev. It was also shown there that bounds of the norm of this projection operator can be calculated in terms of the usual interpolating projection. These results will overcome the second problem. For satisfaction of the conditions required by the theory in [4] and [5], using the analysis in [6] this will be straightforward if we prove that $T D^{-1}$ is a compact operator. That is guaranteed by Kolomogorov and Fomin [7] since $T D^{-1}$ is an integral operator.

Now replacing $D^{m}$ by $D$ and following the same analysis in [1] and [2] we reach smilar expressions for the error bounds with slight computational modifications in certain terms. This is shown in the next section.

\section{EXPRESSIONS FOR THE ERROR BOUNDS WITH THE NEW SPLITTING \\ OF THE DIFFERENTIAL OPERATOR}

If we refer to the bounds presented in [1] and [2], then the bounds with the new splitting will have exactly the same expressions with the following modifications in computation of the following terms:

1. The integral operator $K$ which stands for $T D^{m-1}$ in [1] and [2] will be replaced by $T D^{-1}$;

2. The projection operator $\phi_{n q}$ will be replaced by $\phi_{n q}^{*}$;

3. The collocation matrix $Q_{n q}^{(m)}$ which relates to the highest derivative of the solution and is denoted in [1] by $W_{n}$ will be replaced by the matrix $Q_{n q}^{*}$ where $Q_{n q}^{*}=Q_{n q}^{m}+\lambda_{m-1} Q_{n q}^{m-1}+\ldots+\lambda_{0} Q_{n q}$ and $Q_{n q}^{k}$ relates to the $k$ th derivative of the solution.

4. The subscript $n$ refers to the number of partitions and $q$ refers to the number of collocation points. For global case $n=1$, then the expression of the error bounds with this new splitting and notation will be

$$
\left\|(D-T)^{-1}\right\| \leq P_{d}=\frac{\left\|D^{-1}\right\| \sum_{i=0}^{d}\left\|\left(T D^{-1}\right)^{2}\right\|+\left\|\phi_{n q}^{*}\right\|\left\|Q_{n q}\right\|\left\|\phi_{n q}^{*}\left(T D^{-1}\right)^{d+1}\right\|}{1-\delta_{n q}^{d}}
$$

if

$$
\begin{gathered}
\delta_{n q}^{d}<\left\|\left(\phi_{n q}^{*}-1\right)\left(T D^{-1}\right)^{d+1}\right\|+\left\|\left(\phi_{n q}^{*}-1\right)\left(T D^{-1}\right) \phi_{n q}^{*}\right\|\left\|Q_{n q}^{*}\right\|\left\|\left(T D^{-1}\right)^{d+1}\right\| \leq 1 \\
d=0,1,2, \ldots
\end{gathered}
$$

and

$$
\left\|(D-T)^{-1}\right\| \leq A_{d}=\frac{\left\|D^{-1}\right\| \sum_{i=0}^{d}\left\|\left(T D^{-1}\right)^{i}\right\|\left\|D^{-1} T \phi_{n q}^{*}\right\|\left\|Q_{n q}\right\|\left\|\left(T D^{-1}\right)^{d}\right\|}{1-\triangle_{n}^{d}}
$$

if

$$
\triangle_{n}^{d}<\left\|\left(T D^{-1}\right)\right\|\left\|\left(\phi_{n q}^{*}-1\right)\left(T D^{-1}\right)^{d}\right\|+\left\|\left(T D^{-1}\right)\right\|\left\|\left(\phi_{n q}^{*}-1\right)\left(T D^{-1}\right) \phi_{n q}^{*}\right\|\left\|Q_{n q}^{*}\right\|\left\|\left(T D^{-1}\right)^{d}\right\| \leq 1
$$

$$
d=1,2, \ldots .
$$


Since the main purpose of this paper is to study the problem of applicability we are not going to compare the closeness of these new bounds. However, we don't expect to have any significant changes, especially with the bounds using matrices related directly to the solution as they will not be affected by the new splitting. But for the applicability conditions we expect significant changes since all modified terms are involved.

\section{NUMERICAL APPLICATIONS}

In our numerical application we will consider the simple second order case

$$
x^{\prime \prime}(t)+p_{i}(t) x^{\prime}(t)+p_{0}(t) x(t)=y(t), x( \pm 1)=0
$$

with $D$ taking the simples form

$$
D x=x^{\prime \prime}+\lambda_{0} x,
$$

that is, the coefficient of $x^{\prime}$ is taken zero. Obviously, if $p_{l(t)} \neq 0$, then the inclusion of that parameter is expected to give better results. Generalization to higher order equations and more complicated $D$ is straightforward but a bit tedious.

The test problems will be the same ones considered in [1] and [2].

Problem (1) $\quad x^{\prime \prime}+\alpha\left(1+t^{2}\right) x=y \quad x( \pm t)=0$

Problem (2) $\quad x^{\prime \prime}-\alpha x=y \quad x( \pm 1)=0$

Problem (3) $\quad x^{\prime \prime}-\frac{2 \alpha x}{(t+5)^{2}}=y \quad x( \pm 1)=0$

Problem (4) $\quad x^{\prime \prime}+\frac{2 \alpha x}{t+3}-\frac{2 \alpha x}{(t+3)^{2}}=y \quad x( \pm 1)=0$.

The parameter $\alpha$ is included to vary the smoothness of the problem. Problem 2 will be neglected because it is a trivial case with the above $D . \lambda_{2}$ is firstly chosen the one point best approximation of $p_{t}(t)$. That is, $\lambda_{2}=\frac{1}{2}\left\{\max _{s}\left|p_{i}(s)\right|+\min _{s}\left|p_{\imath}(s)\right|\right\}$.

In table (1) we present bounds for $\left\|T D^{-1}\right\|$ and $\left\|\left(T D^{m}\right)^{-1}\right\|$ and the norms of the matrices related to $D^{m}$ and $D$ for $n=1, q=5$ and $n=1, q=20$. In comparing those bounds we notice the following:

(i) There are some odd values in problem (1) (values with *) where the new splitting gives larger values. The easiest practical way of avoiding these nearly singular cases is to consider other values of $\lambda$ nearby and to choose the best of them, as shown later.

(ii) For problem (3) huge reductions were achieved. That is because $\lambda$ is negative and hence $T D^{-1}$ is well behaved, the function $p_{0}(t)=\frac{-2 \alpha}{(t+5)^{2}}$ does not vary much and can be well approximated by a constant and $p_{1}(t)=0$.

(iii) For problem (4) all values are reduced but the reductions are not as in problem (3) since here $p_{1}(t) \neq 0$. Obviously, if $D$ includes an approximation of $p_{1}$ then similar reductions are expected.

(iv) For most of the cases the reductions increase with $\alpha$, which indicates better results with less smooth problems.

In comparing the norms of the collocation matrices related to $D$ and $D^{m}, Q_{n q}^{*}$ and $W_{n q}$ respectively, we note the following in table (1).

(v)For problem (1) $Q_{n q}^{*}<W_{n q}$ for every value of $\alpha=1,2$ where $\left\|T D^{-1}\right\|$ was shown in (i) to be relatively large. The explanation of this may be due to singularity of $D$ at $\alpha=2,18$ which will affect the values of $W_{n q}$ more than $Q_{n q}^{*}$.

(vi) For problem (3) $Q_{n q}^{*} \ll W_{n q}$ as expected from (ii).

(vii) For problem (4) $Q_{n q}^{*}$ is almost similar to $W_{n q}$. This is probably due to the dominate of the derivative term.

Table (2) compares the smallest number of points required for the applicability of bounds with the original and the modified splittings. That is the bounds $P_{d}$ and $A_{d}$ given by (3.1) and (3.2) and the corresponding bounds described in [1] and [2]. 
We note here the following:

(viii) For problem (1) as expected from (i) and (v) we see good improvements with $\alpha=0.5$ and $\alpha=1$ but no improvement with $\alpha=2$. This case will be reconsidered.

(ix) For problem (3) huge improvements were achieved, especially with $\alpha=100$ as expected from (ii) and (vi).

(x) For problem (4), there are also good improvements as expected from (iii) and (vii).

In table (3) the bounds for $\left\|T D^{-1}\right\|$ are considered with other values of $\lambda$ for problem (1), $\alpha=2$. We see that the bounds take their minimum around $\lambda=1$. The applicability is tested in table (4) with $\lambda=0.5,1,1.25$. We observe that the best applicability occurs for $\lambda=1$ where $\left\|T D^{-1}\right\|$ takes its minimum which supports our method in dealing with the choice of $\lambda$ values.

Table (1)

Comparison of bounds on integral operators and the norms of the related matrices

\begin{tabular}{|c|c|c|c|c|c|c|c|}
\hline \multicolumn{2}{|c|}{ Problem } & \multirow[b]{2}{*}{$\left\|T D^{-1}\right\|$} & \multirow[b]{2}{*}{$\left\|T D^{m^{-1}}\right\|$} & \multirow[b]{2}{*}{ W5 } & \multirow[b]{2}{*}{$Q_{5}^{*}$} & \multirow[b]{2}{*}{$W_{20}$} & \multirow[b]{2}{*}{$Q_{20}^{*}$} \\
\hline$a$ & $\lambda$ & & & & & & \\
\hline \multicolumn{8}{|l|}{1} \\
\hline .5 & .75 & 0.1812 & 0.2500 & 1.3258 & 1.0122 & 1.3257 & 1.1136 \\
\hline 1 & 1.5 & 0.6494 & 0.5000 & 1.9318 & 1.3572 & 1.9318 & 1.3543 \\
\hline 2 & 3 & 2.4064 & 1.000 & 13.4358 & 5.678 & 13.4934 & 5.6359 \\
\hline 100 & 150 & 8.0191 & 50.000 & 526.872 & 112.6927 & 26.2084 & 7.3665 \\
\hline \multicolumn{8}{|l|}{3} \\
\hline .5 & -0.04514 & 0.0045 & 0.03125 & 1.0025 & 1.0042 & 1.01409 & 1.0045 \\
\hline 1 & -.09028 & 0.0088 & 0.0625 & 1.0048 & 1.0027 & 1.0283 & 1.0144 \\
\hline 2 & -.8056 & 0.0171 & 0.1250 & 1.0089 & 1.0060 & 1.0546 & 1.0278 \\
\hline 100 & 9.028 & 0.2058 & 6.250 & 1.475 & 1.2439 & 1.4831 & 1.245 \\
\hline \multicolumn{8}{|l|}{4} \\
\hline .5 & -0.15625 & 0.4755 & 0.6250 & 1.4935 & 1.5017 & 1.5515 & 1.552 \\
\hline 1 & -0.3125 & 0.9074 & 1.250 & 2.0520 & 2.0659 & 2.2038 & 2.2043 \\
\hline 2 & -0.625 & 1.6665 & 2.500 & 3.3172 & 3.337 & 3.7425 & 3.7439 \\
\hline 100 & -312.5 & 17.888 & 125.0 & 32.1639 & 34124 & 148.5813 & 148.7529 \\
\hline
\end{tabular}

Table (2)

Comparison of applicability conditions

(The smallest number of collocation points required for applicability)

\begin{tabular}{|c|c|c|c|c|c|c|c|c|c|}
\hline \multicolumn{2}{|c|}{ Problem } & \multicolumn{2}{|c|}{$\begin{array}{c}p_{0} \\
\text { original modified }\end{array}$} & \multicolumn{2}{|c|}{$\begin{array}{c}p_{1} \\
\text { original } \\
\text { modified }\end{array}$} & \multicolumn{2}{|c|}{$\begin{array}{c}A_{1} \\
\text { original modified }\end{array}$} & \multicolumn{2}{|c|}{$\begin{array}{c}A_{2} \\
\text { original modified } \\
\end{array}$} \\
\hline & & & & & & & & & \\
\hline .5 & 0.75 & 19 & 4 & 8 & 4 & 3 & 2 & 3 & 2 \\
\hline 1 & 1.5 & 82 & 35 & 22 & 19 & 36 & 18 & 9 & 2 \\
\hline 2 & 3 & $>120$ & $>120$ & $>120$ & $>120$ & $>120$ & $>120$ & $>120$ & $>120$ \\
\hline 100 & 150 & $>120$ & $>120$ & $>120$ & $>120$ & $>120$ & $>120$ & $>120$ & $>120$ \\
\hline 3 & & & & & & & & & \\
\hline & -004514 & 2 & 2 & 2 & 2 & 2 & 2 & 2 & 2 \\
\hline 1 & -.09028 & 2 & 2 & 2 & 2 & 2 & 2 & 2 & 2 \\
\hline 2 & -.18056 & 2 & 2 & 2 & 2 & 2 & 2 & 2 & 2 \\
\hline 100 & -9.028 & $>120$ & 22 & $>120$ & 10 & $>120$ & 4 & $>120$ & 3 \\
\hline 4 & & & & & & & & & \\
\hline .5 & -0.15625 & 33 & 17 & 12 & 6 & 13 & 5 & 6 & 4 \\
\hline 1 & -0.3125 & $>120$ & 97 & 38 & 22 & $>120$ & 65 & 24 & 16 \\
\hline 2 & -0.625 & $>120$ & $>120$ & $>120$ & 74 & $>120$ & $>120$ & $>120$ & 77 \\
\hline 100 & -312.5 & $>120$ & $>120$ & $>120$ & $>120$ & $>120$ & $>120$ & $>120$ & $>120$ \\
\hline
\end{tabular}


Table (3)

Bound on $\left\|T D^{-1}\right\|$ with other values of $\lambda$ near 3 for problem (1), $a=2$

\begin{tabular}{|l|l|l|l|l|l|l|l|l|l|}
\hline$\lambda$ & 2.75 & 2.5 & 3.25 & 2 & 1.75 & 1.5 & 1.25 & 1 & 0.75 \\
\hline$\left\|T D^{-1}\right\|$ & 3.393 & 19.538 & 1.9816 & 1.2342 & 1.0385 & 0.9657 & .9401 & 0.9365 & 0.9459 \\
\hline
\end{tabular}

Table (4)

The applicability for values of $\lambda$ with small bounds of $\left\|T D^{-1}\right\|$ for problem (1), $a=2$

\begin{tabular}{|l|l|l|l|l|}
\hline$\lambda$ & $p_{0}$ & $p_{1}$ & $A_{1}$ & $A_{2}$ \\
\hline .5 & $>120$ & 95 & $>120$ & 61 \\
\hline 1 & $>120$ & 85 & $>120$ & 48 \\
\hline 1.25 & $>120$ & 87 & $>120$ & 49 \\
\hline
\end{tabular}

\section{CONCLUSION}

It is shown in this paper that extension of the principal part of the differential operator to be a linear combination with constant parameters of all the derivatives can be one practical solution to the problem of applicability of the error bounds derived in [1] and [2].

The numerical results have shown that significant improvements in the applicability are achieved with this new extension. More investigation in the method of choosing the parameters or in looking for other principal part extensions may be needed for further improvements. One option could be to consider the equation in the form

$$
\left(I-D^{m^{-1}} T\right) x=D^{m^{-1}} y
$$

and to investigate for satisfaction of the theory and look for practical bounds for $\left\|D^{m^{-1}} T\right\|$.

\section{REFERENCES}

[1] CRUICKSHENK, D.M. and WRIGHT, K., Computable error bounds for polynomial collocation methods, SIAM J. Num. Anal. 15 (1978), 134-151.

[2] AHMED, A.H., Computable error bound for collocation methods, Internat. J. Math. \& Math. Sci. 18 (1995), 89-96.

[3] AHMED, A.H., Collocation algorithms and error analysis for approximate solutions of ordinary differential equations, Ph.D. thesis, University of Newcastle Upon Tyne (1981).

[4] KANTOROVICH, L.V. and AKIIOV, G.P., Functional Analysis in Normed Spaces, Pergaman Press, New York (1964).

[5] ANSELONE, P.M., Collectively Compact Operator Approximation Theory, Prentice Hall, Englewood Cliffs, NJ (1971).

[6] AHMED, A.H., Asymptotic properties of collocation projection norms, Computer Math. Applic. 19, 4 (1990), 45-50.

[7] KOLOMOGOROV, A.N. and FOMIN, S.V., Functional Analysis, Vol. 1, Graylock, Rochester, NY (1957) 


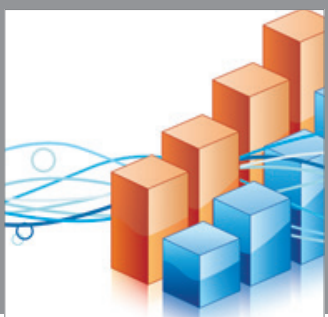

Advances in

Operations Research

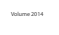

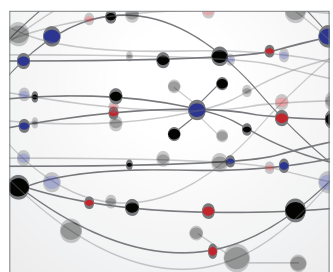

\section{The Scientific} World Journal
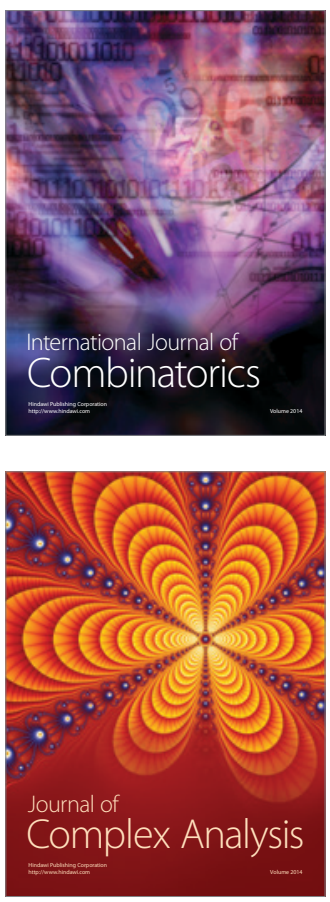

International Journal of

Mathematics and

Mathematical

Sciences
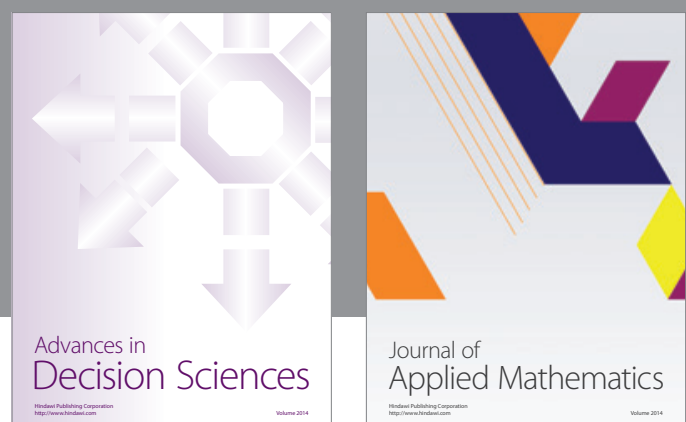

Journal of

Applied Mathematics
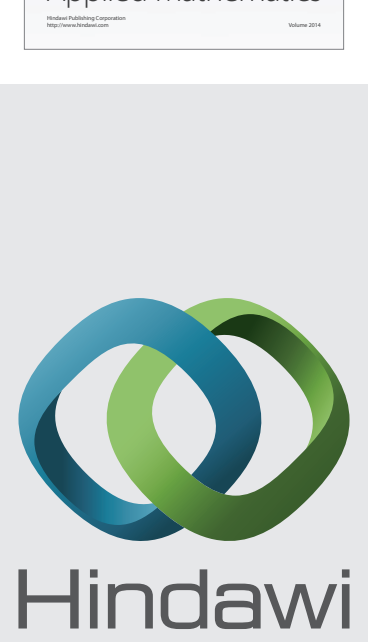

Submit your manuscripts at http://www.hindawi.com
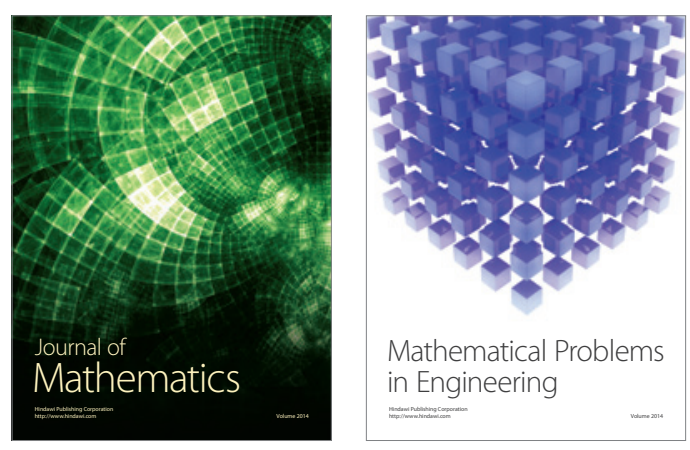

Mathematical Problems in Engineering
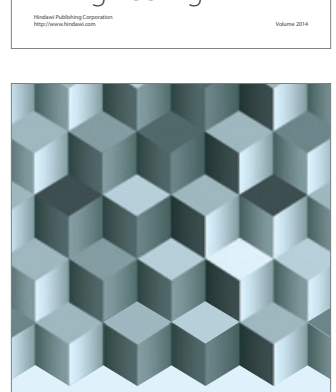

Journal of

Function Spaces
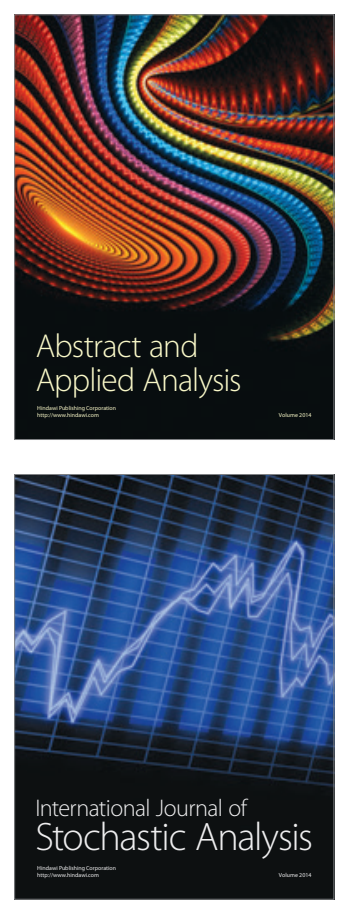

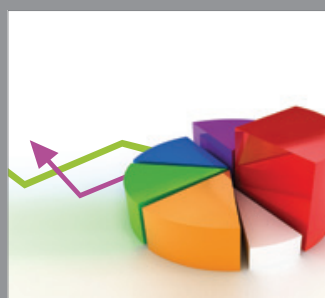

ournal of

Probability and Statistics

Promensencen
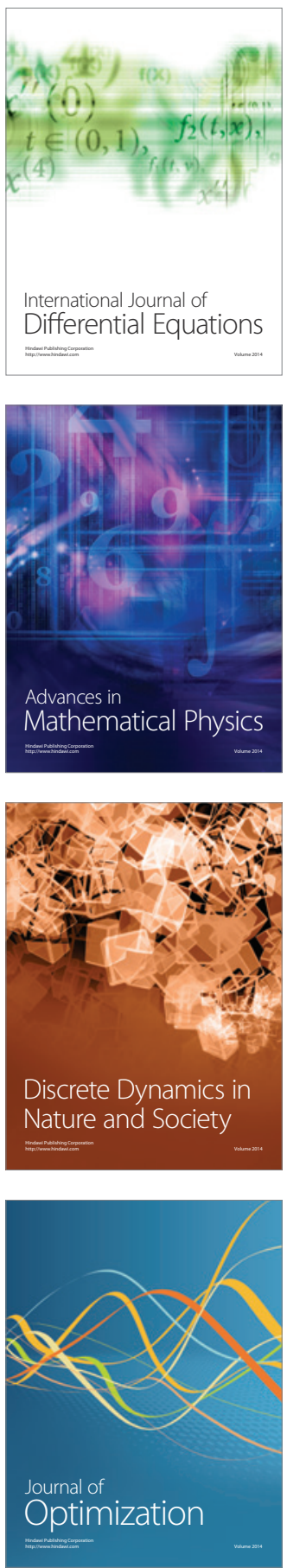